\title{
Preface (Vol 35, Issue 6,7,8)
}

\author{
Nadia Magnenat-Thalmann ${ }^{1}$
}

(c) Springer-Verlag GmbH Germany, part of Springer Nature 2019

Welcome to the special issue of the 36th Computer Graphics International (CGI 2019) conference. CGI is one of the oldest international conferences in computer graphics in the world. It is the official conference of the Computer Graphics Society (CGS), a long-standing international computer graphics organization. CGI and CGS were initiated by Professor Tosiyasu L. Kunii from the University of Tokyo in 1983. Since then, the CGI conference has been held annually in many different countries across the world and gained a reputation as one of the key conferences for researchers and practitioners to share their achievements and discover the latest advances in computer graphics. This year, CGI 2019 is organized by CGS and the University of Calgary and is being held in Calgary, Alberta, Canada, from June 17-20, 2019. CGI 2019 is in cooperation with ACM/SIGGRAPH and EUROGRAPHICS.

This special issue of the Visual Computer is composed of the 33 best papers from CGI 2019. We received 162 submissions for the Visual Computer track from 31 countries and regions, with an acceptance ratio of about $21.6 \%$. To ensure the highest quality of publications, each paper has been reviewed by at least three experts in the field. The International Program Committee is composed of 132 members plus 93 external sub-reviewers invited by the IPC.

We would like to express our deepest gratitude to all the IPC members and external reviewers who have provided high-quality reviews timely. We would also like to thank all the authors for contributing to the conference by submitting their work. Our special appreciation goes to the organizing committee who has contributed to the success of the CGI 2019.

Nadia Magnenat-Thalmann

thalmann@miralab.ch

1 University of Geneva, Geneva, Switzerland

\begin{tabular}{ll}
\hline Conference chairs & Program chairs \\
\hline $\begin{array}{l}\text { Marina L. Gavrilova } \\
\text { University of Calgary, Canada }\end{array}$ & $\begin{array}{l}\text { Nadia Magnenat-Thalmann } \\
\text { University of Geneva, } \\
\text { Switzerland }\end{array}$ \\
$\begin{array}{l}\text { Hiroshi Ishikawa } \\
\text { Waseda University, Japan } \\
\text { http://www.cgs-network.org/ } \\
\text { cgi19/ }\end{array}$ & Jian Chang \\
\hline
\end{tabular}

\section{List of Articles in this special issue}

The selected 33 papers of CGI 2019 are organized in the following 7 sections, covering the fundamentals as well as the most advanced research topics in computer graphics as listed below.

$3 D$ Reconstruction: The first section is on $3 \mathrm{D}$ reconstruction and consists of 2 papers.

1. "Expressive Facial Style Transfer for Personalized Memes Mimic" by Yanlong Tang, Ruofeng Tang from Zhejiang University, China, Xiaoguang Han from The Chinese University of Hong Kong (Shenzhen), Yue Li from University of Pennsylvania, USA, and Liqian Ma from Beijing Kuaishou Technology Ltd., China.

2. "Joint Bilateral Propagation Upsampling for Unstructured Multi-View Stereo" by Mengqiang Wei, Chunxia Xiao from Wuhan University, China, and Qingan Yan from JD.com American Technologies Corporation, USA.

Rendering Techniques: The second section is on rendering techniques and consists of 3 papers.

1. "Image-Based Translucency Transfer through Correlation of Multi-Scale Spatial Color Distribution" by Hideki Todo from Chuo Gakuin University, Japan, Tatsuya Yatagawa from Waseda University, Japan, Masataka Sawayama from NTT Communication Science Laboratories, Japan, Yoshinori Dobashi and Masanori Kakimoto from Dwango CG research, Japan. 
2. "Stereoscopic Image Stitching with Rectangular Boundaries" by Yun Zhang from Communication University of Zhejiang, China, Yu-Kun Lai from Cardiff University, UK, and Fang-Lue Zhang from Victoria University of Wellington, New Zealand.

3. "Multiresolution Visualization of Massive Black Oil Reservoir Models" by Frederico Abraham and Waldemar Celes from Pontifical Catholic University of Rio de Janeiro, Brazil.

Virtual Reality and Augmented Reality: The third section focuses on virtual and augmented reality with 4 papers.

1. "Interactive Animation Generation of Virtual Characters Using Single RGB-D Camera" by Ning Kang, Junxuan Bai, Junjun Pan from Beihang University, China, and Hong Qin from Stony Brook University (SUNY Stony Brook), USA.

2. "Real-time Simulation of Electrocautery Procedure using Mesh-free Methods in Laparoscopic Cholecystectomy" by Junjun Pan, Yuhan Yang, Yang Gao from Beihang University, China, Hong Qin from Stony Brook University (SUNY Stony Brook), USA, and Yaqing Si from The First Affiliated Hospital of Zhengzhou University, China.

3. "DeepLight: Light Source Estimation for Augmented Reality Using Deep Learning” by Peter Kán and Hannes Kaufmann from TU Wien, Austria.

4. "AniCode: Authoring Coded Artifacts for Network-Free Personalized Animations" by Zeyu Wang, Shiyu Qiu, Qingyang Chen, Alexander Ringlein, Julie Dorsey and Holly Rushmeier from Yale University, USA.

Geometric Modelling, Geometric Computing and Shape and Surface Modelling: The fourth section consists of 6 papers.

1. "Acquiring periodic tilings of regular polygons from images” by José Ezequiel Soto Sánchez, Luiz Henrique de Figueiredo from IMPA, Brazil, and Asla Medeiros E Sá from FGV EMAp, Brazil.

2. "Image-driven Unsupervised 3D Model Cosegmentation" by Juncheng Liu, Zhouhui Lian, Jianguo Xiao from Peking University, China, Paul L. Rosin, Xianfang Sun from Cardiff University, UK.

3. "Minkowski Sum Computation for Planar Freeform Geometric Models using G1-Biarc Approximation and Interior Disks" by Sangjun Han, Myung-Soo Kim from Seoul National University, South Korea, Seung-Hyun Yoon from Dongguk University, South Korea, and Gershon Elber from Technion, Israel.

4. "Huber-L1 Based Non-Isometric Surface Registration" by Tao Jiang, Xiaosong Yang, Jianjun Zhang, Feng Tian,
Shuang Liu, Nan Xiang, from Bournemouth University, UK, and Kun Qian from King's College London, UK.

5. "A Lightweight Methodology of 3D Printed Objects utilizing Multi-Scale Porous Structures" by Jiangbei Hu, Shengfa Wang, Yi Wang, Fengqi Li and Zhongxuan Luo from Dalian University of Technology, China.

6. "Generating Signed Distance Fields on the GPU with Ray Maps" by Bastian Krayer and Stefan Müller from University Koblenz-Landau, Germany.

Visual Analytics, Image Processing and Pattern Recognition: The fifth section consists of 10 papers about visual analytics, image processing and pattern recognition.

1. "Retrieval of spatial-temporal motion topics from 3D skeleton data" using Qianhui Men and Howard Leung from City University of Hong Kong, Hong Kong.

2. "Weighted Superpixel Segmentation" by Xin Qian, Xuemei Li and Caiming Zhang from Shandong University, China.

3. "Deep Binocular Tone Mapping" by Zhuming Zhang, Chu Han, Xinghong Hu, Tien-Tsin Wong from The Chinese University of Hong Kong, Hong Kong, Shengfeng He from South China University of Technology, China, Xueting Liu from Caritas Institute of Higher Education, Hong Kong, and Haichao Zhu from Rokid Corporation Ltd.

4. "TimeCluster: Dimension Reduction of Temporal Data for Visual Analytics" by Mohammed Ali, Mark Jones, Xianghua Xie from Swansea University, UK, and Mark Williams from University of South Wales, UK.

5. "Depth-aware Image Vectorization and Editing" by Shufang Lu, Xuefeng Ding, Fei Gao, Jiazhou Chen from Zhejiang University of Technology, China, Wei Jiang from University of Victoria, Canada, Craig S. Kaplan from University of Waterloo, Canada, and Xiaogang Jin from Zhejiang University, China.

6. "Density-based label placement" by Antoine Lhuillier, Daniel Weiskopf from University of Stuttgart, Germany, and Mereke Van Garderen from University of Konstanz, Germany.

7. "Processing images for red-green dichromats compensation via naturalness- and information-preservation considered recoloring" by Zhenyang Zhu, Masahiro Toyoura, Kentaro Go, Kenji Kashiwagi, Xiaoyang Mao from University of Yamanashi, Japan, and Issei Fujishiro from Keio University, Japan.

8. "The Visual SuperTree: Similarity-based Multi-Scale Visualization" by Renato Rodrigues Oliveira da Silva, Carlos Elias Arminio Zampieri, Fabio Pavan Rolli, Rosane Minghim from University of Sao Paulo, Brazil, José Gustavo Souza Paiva from Federal University of 
Uberlandia, Brazil, and Guilherme Pimentel Telles from University of Campinas, Brazil.

9. "Blind Image Deblurring with Reinforced Use of Edges" by Feng Qiu, Fei Hou and Wencheng Wang from Chinese Academy of Sciences, China.

10. "Effective Shadow Removal via Multi-scale Image Decomposition" by Ling Zhang, Xiaolong Zhang from Wuhan University of Science and Technology, China, Qingan Yan from JD.com American Technologies Corporation, USA, and Yao Zhu and Chunxia Xiao from Wuhan University, China.

Computer Animation: The sixth section consists of 4 papers focusing on computer animation.

1. "Building Anatomically Realistic Jaw Kinematics Model from Data" by Wenwu Yang from Zhejiang Gongshang University, China, Nathan Marshak, Srikumar Ramalingam, Ladislav Kavan from University of Utah, USA, and Daniel Sýkora from Czech Technical University in Prague, Czech Republic.

2. "CrowEst: A Method for Estimating (and not Simulating) Crowd Evacuation Parameters in Generic Environments" by Estêvão Testa, Rodrigo Barros and Soraia Musse from Pontifical Catholic University of Rio Grande do Sul, Brazil.

3. "Precomputed Optimal One-Hop Motion Transition for Responsive Character Animation" by Yuki Koyama and Masataka Goto from National Institute of Advanced Industrial Science and Technology, Japan.

4. "Efficient Convolutional Hierarchical Autoencoder for human motion prediction" by Yanran Li, Xiaosong Yang, Sebastian Iulian Poiana, Ehtzaz Chaudhry, Jianjun Zhang from Bournemouth University, UK, Zhao Wang from Nanjing Institute of Advanced Artificial Intelligence, China, and Meili Wang from Northwest A\&F University, China.

Machine Learning for Graphics: The seventh section focuses on machine learning for graphics with 4 papers.

1. "Stylistic Scene Enhancement GAN: Mixed Stylistic Enhancement Generation for 3D Indoor Scenes" by Suiyun Zhang, Hui Zhang from Tsinghua University, China, Zhizhong Han, Matthias Zwicker from University of Maryland, USA, and Yu-Kun Lai from Cardiff University, UK.

2. "Deep Similarity Network Fusion for 3D Shape Classification" by Lorenzo Luciano from Amazon Web Services, USA, and Abdessamad Ben Hamza from Concordia University, Canada.

3. "Example-based Rapid Generation of Vegetation on Terrain via CNN-based Distribution Learning" by Jian
Zhang, Changbo Wang, Chen Li from East China Normal University, China, and Hong Qin from Stony Brook University (SUNY Stony Brook), USA.

4. "Intelligent Chinese Calligraphy Beautification from Handwritten Characters for Robotic Writing" by Xinyue Zhang, Yuanhao Li, Zhiyi Zhang, Shaojun Hu from Northwest A\&F University, China, and Kouichi Konno from Iwate University, Japan.

\section{International program committee}

- Usman Alim, University of Calgary, Canada

- Ryoichi Ando, Kyushu University, Japan

- Marco Attene, CNR IMATI, Italy

- Melinos Averkiou, University of Cyprus, Cyprus

- Selim Balcisoy, Sabanci University, Turkey

- Loic Barthe, IRIT-Université de Toulouse, France

- Jan Bender, RWTH Aachen, Germany

- Bedrich Benes, Purdue University, USA

- Silvia Biasotti, CNR IMATI, Italy

- Nicolas Bonneel, CNRS-Univ. Lyon, France

- Stefan Bruckner, University of Bergen, Norway

- Yiyu Cai, Nanyang Technological University, Singapore

- Tolga Capin, TED University, Turkey

- Jian Chang, Bournemouth University, UK

- Parag Chaudhuri, Indian Institute of Technology, Bombay, India

- Li Chen, School of Software, Tsinghua University, China

- Falai Chen, University of Science and Technology of China, China

- Jie Chen, University of Oulu, Finland

- Marc Christie, University of Rennes 1, France

- David Coeurjolly, CNRS, Université de Lyon, France

- Frederic Cordier, Universite de Haute Alsace, France

- Remi Cozot, IRISA, University of Rennes 1, France

- Zhigang Deng, University of Houston, USA

- Julie Digne, CNRS, Université de Lyon, France

- Jean-Michel Dischler, University of Strasbourg, France

- Yoshinori Dobashi, Hokkaido University, Japan

- Yuki Endo, Toyohashi University of Technology, Japan

- Parris Egbert, Brigham Young University, USA

- Petros Faloutsos, York University, EECS, Canada

- Jieqing Feng, Zhejiang University, China

- Ioannis Fudos, University of Ioannina, Greece

- Issei Fujishiro, Keio University, Japan

- Xifeng Gao, Florida State University, USA

- Marina Gavrilova, University of Calgary, Canada

- Enrico Gobbetti, CRS4 Visual Computing, Italy

- Laurent Grisoni, University of Lille 1, France

- Roberto Grosso, Friedrich-Alexander-Universität Erlangen-Nürnberg, Germany

- Shihui Guo, Xiamen University, China 
- Stefan Guthe, TU Darmstadt, Germany

- Atsushi Hashimoto, Kyoto University, Japan

- Hui Huang, Shenzhen University, China, China

- Hua Huang, Beijing Institute of Technology, China

- Satoshi Iizuka, Waseda University, Japan

- Yuki Igarashi, Meiji University, Japan

- Kei Iwasaki, Wakayama University, Japan

- Xiaogang Jin, Zhejiang University, China

- Prem Kalra, IIT Delhi, India

- Takashi Kanai, University of Tokyo, Japan

- Yoshihiro Kanamori, University of Tsukuba, Japan

- Hyungseok Kim, Konkuk University, South Korea

- Jinman Kim, University of Sydney, Australia

- Stefanos Kollias, Professor, Greece

- Yuki Koyama, National Institute of Advanced Industrial Science and Technology (AIST), Japan

- Barbora Kozlikova, Masaryk University, Czech Republic

- Arjan Kuijper, Fraunhofer IGD \& TU Darmstadt, Germany

- Tsz Ho Kwok, Concordia University, Canada

- Yu-Kun Lai, Cardiff University, UK

- Guillaume Lavoue, INSA-CNRS, Lyon, France

- Ligang Liu, University of Science and Technology of China, China

- Nadia Magnenat-Thalmann, NTU Singapore \& MIRALab-University of Geneva, Switzerland

- Xiaoyang Mao, University of Yamanashi, Japan

- Kresimir Matkovic, VRVis Research Center, Austria

- Jianyuan Min, Google, USA

- Bochang Moon, Gwangju Institute of Science and Technology, South Korea

- Shigeo Morishima, Waseda University, Japan

- Michela Mortara, CNR IMATI, Italy

- Sudhir Mudur, Concordia University, Canada

- Heinrich Mueller, University of Dortmund, Germany

- Tomohiko Mukai, Tokyo Metropolitan University, Japan

- Soraia Musse, Pontificia Universidade Catolica do Roi Grande do Sul, PUCRS, Brazil, Brazil

- Yukie Nagai, The University of Tokyo, Japan

- Masayuki Nakajima, Uppsala University, Sweden

- Junyong Noh, KAIST, South Korea

- Makoto Okabe, Shizuoka University, Japan

- Masaki Oshita, Kyushu Institute of Technology, Japan

- Zhigeng Pan, Hangzhou Normal University, China

- Daniele Panozzo, NYU, USA

- George Papagiannakis, University of Crete, Greece

- Alexander Pasko, Bournemouth University, UK

- Giuseppe Patanè, CNR IMATI, Italy

- Konrad Polthier, Freie Universität Berlin, Germany

- Luciana P. Nedel, Universidade Federal do Rio Grande do Sul, Brazil

- Nicolas Pronost, Université Claude Bernard Lyon 1, France
- Renata Georgia Raidou, Vienna University of Tech, Austria

- Holly Rushmeier, Yale, USA

- Filip Sadlo, Heidelberg University, Germany

- Gerik Scheuermann, University of Leipzig, Germany

- Teseo Schneider, New York University, USA

- Michael Schwarzler, Delft University of Technology, Netherlands

- Hyewon Seo, ICube-University of Strasbourg, France

- Ari Shapiro, USC Institute for Creative Technologies, USA

- Ehud Sharlin, University of Calgary, Canada

- Ojaswa Sharma, Institute of Information Technology, Delhi, India

- Jianbing Shen, School of Computer Science, Beijing Institute of Technology, China

- Yun Sheng, East China Normal University, China

- Edgar Simo-Serra, Waseda University, Japan

- Claudio T. Silva, New York University, USA

- Oh-young Song, Sejong University, South Korea

- Alexei Sourin, Nanyang Technological University, Singapore

- Olga Sourina, Nanyang Technological University, Singapore

- Michela Spagnuolo, CNR IMATI, Italy

- Suguru Saito, Tokyo Institute of Technology, Japan

- Hanqiu Sun, The Chinese University of Hong Kong, Hong Kong

- Kenshi Takayama, National Institute of Informatics, Japan

- Daniel Thalmann, EPFL, Switzerland

- Hideki Todo, Chuo Gakuin University, Japan

- Ruofeng Tong, Zhejiang University, China

- Masahiro Toyoura, University of Yamanashi, Japan

- Amir Vaxman, Utrecht University, Netherlands

- Marcelo Walter, UFRGS, Brazil

- Charlie Wang, The Chinese University of Hong Kong, China

- Hongcheng Wang, Comcast Labs, USA

- Meili Wang, Northwest A\&F University, China

- Wencheng Wang, Chinese Academy Of Science, China

- Franz-Erich Wolter, Gottfried Wilhelm Leibniz University of Hannover, Germany

- Tien-Tsin Wong, CUHK, Hong Kong

- Enhua Wu, University of Macau \& ISCAS, China

- Kai (Kevin) Xu, National University of Defense Technology, China

- Xiaosong Yang, Bournemouth University, UK

- Junfeng Yao, Xiamen University, China

- Tatsuya Yatagawa, Waseda University, Japan

- Norimasa Yoshida, Nihon University, Japan

- Lihua You, Bournemouth University, UK

- Yonghao Yue, The University of Tokyo, Japan

- Zerrin Yumak, Utrecht University, Netherlands 
- Xenophon Zabulis, FORTH, Greece

- Jian J. Zhang, Bournemouth University, UK

- Kang Zhang, University of Texas at Dallas, USA

- Jianmin Zheng, Nanyang Technological University, Singapore

- Youyi Zheng, Zhejiang University, China
- Kun Zhou, Zhejiang University, China

- Changqing Zou, University of Maryland, USA

Publisher's Note Springer Nature remains neutral with regard to jurisdictional claims in published maps and institutional affiliations. 